\title{
Improved control of the betatron coupling in the Large Hadron Collider
}

\author{
T. Persson* \\ CERN \& Chalmers University of Technology, 41296 Gothenburg, Sweden
}

R. Tomás

CERN, CH-1211 Geneva 23, Switzerland

(Received 28 March 2014; published 28 May 2014)

\begin{abstract}
The control of the betatron coupling is of importance for safe beam operation in the LHC. In this article we show recent advancements in methods and algorithms to measure and correct coupling. The benefit of using a more precise formula relating the resonance driving term $f_{1001}$ to the $\Delta Q_{\min }$ is presented. The quality of the coupling measurements is increased, with about a factor 3 , by selecting beam position monitor (BPM) pairs with phase advances close to $\frac{\pi}{2}$ and through data cleaning using singular value decomposition with an optimal number of singular values. These improvements are beneficial for the implemented automatic coupling correction, which is based on injection oscillations, presented in the article. Furthermore, a proposed coupling feedback for the LHC is presented. The system will rely on the measurements from BPMs equipped with a new type of high resolution electronics, diode orbit and oscillation, which will be operational when the LHC restarts in 2015. The feedback will combine the coupling measurements from the available BPMs in order to calculate the best correction.
\end{abstract}

PACS numbers: 29.20.D-, 29.27.Ac, 41.75.-i, 41.85.-p

\section{INTRODUCTION}

The LHC design puts very tight requirements for optics distortions [1]. A large effort has been put into understanding and correcting $\beta$-beat, betatron coupling, chromatic coupling, amplitude detuning with ac dipole and nonlinear studies [2-6]. Since the start of the LHC several coupling measurements and corrections have been performed using free and forced [7] betatron oscillations. In [8] the method to calculate the coupling from ac dipole excited turn-by-turn (TbT) data is described. The strong local coupling sources were corrected during the optics commissioning using the method described in [2] and remained constant during the 2012 run [9]. After the commissioning it is, however, still observed that parameters such as the global coupling varies in time. This is an unwanted effect since the coupling disturbs the tune feedback and can push tunes into resonances or simply lead to a reduction in the dynamic aperture [10]. The drift of the global coupling has been mitigated via two orthogonal knobs which are used to control the real and imaginary part of the $C^{-}$[11]. The knobs were changed by the operator in an iterative manner while observing the $\left|C^{-}\right|$measured from a single dedicated high precision pickup, BBQ [12]. This approach is suffering from two drawbacks. First, it is

*tobias.persson@cern.ch

Published by the American Physical Society under the terms of the Creative Commons Attribution 3.0 License. Further distribution of this work must maintain attribution to the author(s) and the published article's title, journal citation, and DOI. only possible to measure the amplitude of the coupling, which means that the operator has to scan the knobs to find the optimal setting. Second, it fully relies on the measurement at a single location by a single BPM. This can be deceiving since correcting the coupling locally does not guarantee that it is minimized globally, plus a single BPM does not allow us to distinguish between the $f_{1001}$ (difference resonance) and the $f_{1010}$ (sum resonance). A natural step as the machine operation matures is to find more reliable and automatized ways to measure and perform these corrections. In this article we focus on the recent advances of the coupling control in the LHC, which derive from improvements in the reconstruction, understanding, and the use of an automatic correction approach to correct the coupling.

There exist different methods to measure the coupling in circular machines. Examples include exciting horizontal steerer while observing vertical orbit [13], closest tune approach [14], fitting injection data to a N-Map [15] or through the use of resonance driving terms (RDTs) [16]. In Relativistic Heavy Ion Collider (RHIC), extensive care has been taken to online control the coupling [17-19].

In this article we will use the RDTs $f_{1001}$ and $f_{1010}$ to describe the coupling. In the LHC the fractional tunes are $Q_{x}=0.28$ and $Q_{y}=0.31$ at injection and $Q_{x}=0.31$ and $Q_{y}=0.32$ at collision and as a consequence the $\left|f_{1001}\right| \gg\left|f_{1010}\right|$ during normal operation. Their relation to the C-matrix is described in [20].

This article describes improvements to the algorithms in calculating the coupling parameters followed by the realization of an automatic coupling correction based on the 
injection oscillations. The coupling is reconstructed locally by the $\sim 500$ TbT BPMs. Finally, we will describe the design of a feedback to control the coupling beyond 2015 . In Sec. II we start with describing a more precise equation relating the $f_{1001}$ to the $C^{-}$. We continue in Sec. III with demonstrating the benefit of selecting two BPMs close to $\frac{\pi}{2}$ when measuring the coupling. The measurement resolution is also increased using a singular value decomposition (SVD) cleaning, which is described in Sec. IV. The automatic coupling correction approach based on injection oscillations, which was used in normal operation in 2012, is demonstrated in Sec. V. The coupling is reconstructed from all BPMs and the paring algorithm ensures that the phase advance is close to the optimal. The method to use the injection oscillations, however, only provides measurements for injection energy. The resolution of the BPMs is not good enough to measure the coupling from acceptable excitations during normal operation with high intensity beams. The diode orbit and oscillation (DOROS) [21] are being developed at CERN and will provide very precise phase and amplitude measurements. The location of the BPMs equipped with DOROS electronics are, however, not optimized for coupling measurements, since their main purposes are to provide very precise orbit and phase measurements close to the interaction points (IPs). As a consequence the phase advance is far from the optimal $\frac{\pi}{2}$. In Sec. VI a feedback based on the combined information from all the BPMs equipped with DOROS electronics is presented.

\section{MORE PRECISE EQUATION FOR $C^{-}$}

The closest the horizontal and vertical tune can approach each other is termed $\Delta Q_{\min }$ and is equal to the $\left|C^{-}\right|$. The RDT $f_{1001}$ is a local property related to the Hamiltonian term $h_{1001}$. A relation of the $f_{1001}$ to the $\left|C^{-}\right|$close to the difference resonance, was described as [22]

$$
\Delta Q_{\min }=\left|C^{-}\right| \approx 4 \Delta \frac{1}{N} \sum_{i=1}^{N}\left|f_{1001 i}\right|,
$$

where $\Delta Q_{\min }$ is the closest the tunes can approach each other, $N$ is the number of BPMs and $\Delta$ is the fractional tune split. A more precise relation was published in [23] but never applied to data. The nomenclature used in this article is different and we therefore derive the relation in the Appendix for clarity. The relation is described as

$$
\Delta Q_{\min }=\left|C^{-}\right|=\left|\frac{4 \Delta}{2 \pi R} \oint d s f_{1001} e^{-i\left(\phi_{x}-\phi_{y}\right)+i s \Delta / R}\right|,
$$

where $R$ is the radius of the machine, $\phi_{x}$ is the horizontal phase, $\phi_{y}$ is the vertical phase, and $s$ is the longitudinal distance. The integral extends over the entire ring but in practice it will only be evaluated at the locations of the

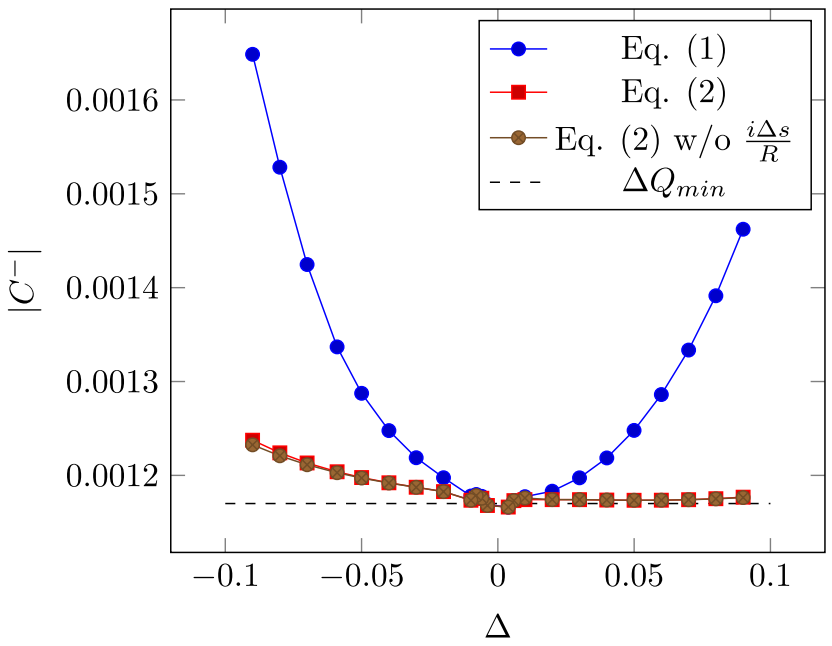

FIG. 1. A comparison of Eqs. (1) and (2) to calculate the $\Delta Q_{\min }$ from $f_{1001} \cdot Q_{y}$ was kept constant at 59.31 while $Q_{x}$ was varied between 64.22 and 64.40. Injection optics for the LHC was used in the simulation.

BPMs. In Fig. 1 the $\left|C^{-}\right|$is calculated from Eqs. (1) and (2). The $\Delta Q_{\min }$ is retrieved by trying to match the tunes as close as possible to each other in methodical accelerator design (MAD) [24]. We observe that the two formulas give almost identical and correct results close to the resonance but Eq. (1) deviates more when the fractional tune split increases. We also observe that the term $i \Delta s / R$ has a negligible effect on the calculated $\left|C^{-}\right|$. This also holds true for the European Synchrotron Radiation Facility (ESRF) booster [25]. The main differences between the formulas can then be interpreted as Eq. (1) is the average of the $\left|f_{1001}\right|$ while Eq. (2) is the absolute value of the average $f_{1001}$.

\section{OPTIMAL PARING OF BPMs}

We reconstruct the $f_{1001}$ and the $f_{1010}$ terms from TbT data [26] using the Courant-Snyder variable [27]

$$
h_{x,-}=\hat{x}-i \hat{p_{x}},
$$

where $\hat{x}$ is the normalized horizontal position and $\hat{p}_{x}$ is the horizontal transverse momentum. The momentum is not a directly measurable quantity with a BPM but needs to be reconstructed using two BPMs. The momentum at the $i$ th $\mathrm{BPM}$ can be written as [22]

$$
\hat{p}_{x i}=\frac{\hat{x}_{i+1}-\hat{x}_{i} \cos \Delta \phi_{x}}{\sin \Delta \phi_{x}},
$$

where $\Delta \phi_{x}$ is the horizontal phase advance between the $i$ th and $(i+1)$ th BPM under the assumption that the region between the two BPMs is free of coupling sources and nonlinearities contributing to the main and the coupling line. Equation (4) indicates that a phase advance of $\frac{\pi}{2}$ is the 

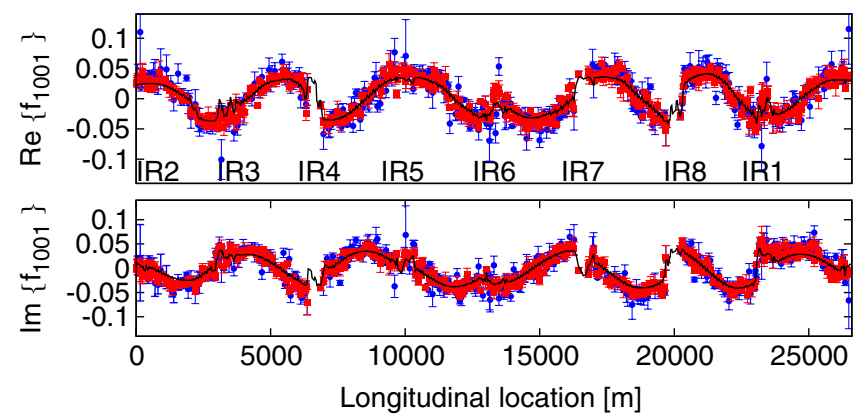

Consecutive BPMs $\longmapsto$ $\pi / 2$ phase advance Model

FIG. 2. Comparison of reconstructing the coupling using consecutive BPMs (blue) and BPMs with close to $\frac{\pi}{2}$ phase advance (red) for simulated data.

optimal to minimize the error since $\sin \Delta \phi_{x}$ reaches its maximum value while $\cos \Delta \phi_{x}$ reaches the minimum when $\Delta \phi_{x}=\frac{\pi}{2}$.

Following this observation we propose to pair each BPM with the one downstream, up to maximum 5 BPMs away, which has the phase advance closest to $\frac{\pi}{2}$. The BPMs used are dual planes TbT BPMs which enables calibrationindependent measurement of the coupling, described in [22].

A simulation is made in order to validate the new improved way of paring the BPMs. A realistic coupling situation is introduced in the MAD [24] model. The particles were tracked for 1000 turns using polymorphic tracking code (PTC) [28] and Gaussian distributed noise with an amplitude of $\sim 40 \%$ of the betatron oscillation is added to the TbT data. This is a realistic noise-to-signal ratio from a measurement based on injection oscillations but a dedicated kick will in general create a larger oscillation and hence the relative noise will be smaller. In total, 3 data sets with 1000 turns each were created, which simulate 3 measurements in the real machine. The multiple data sets were used to calculate the error bars. In Fig. 2 a comparison between the two ways of paring the BPMs is shown together with the model. From Fig. 2 it is

TABLE I. A comparison of the two different BPM paring algorithms. $\sigma_{\text {Consecutive }}$ is the average error bar for BPM pairs with consecutive BPM, $\sigma_{\pi / 2}$ is the average error bar for BPM pairs with phase advance close to $\pi / 2, f_{1001}^{\text {model }}$ is the $f_{1001}$ reconstructed from the model, $f_{1001}^{\pi / 2}$ is the reconstructed $f_{1001}$ from BPM pairs with a phase advance close to $\pi / 2$ and $f_{1001}^{\text {Consecutive }}$ from BPM pairs with consecutive BPM.

\begin{tabular}{|c|c|c|c|c|}
\hline Type & $\sigma_{\text {Consecutive }}$ & $\sigma_{\pi / 2}$ & $\frac{\sigma_{\text {Consecutive }}}{\sigma_{\pi / 2}}$ & 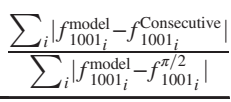 \\
\hline AC-dipole data & 0.00301 & 0.00144 & 2.09 & $\ldots$ \\
\hline Kick data & 0.0023 & 0.000956 & 2.41 & $\cdots$ \\
\hline Simulation & 0.013 & 0.0071 & 1.83 & 1.42 \\
\hline
\end{tabular}

observed that choosing the phase advance closer to $\frac{\pi}{2}$ reduces both the error bars and the deviation from the correct value. The biggest improvements are close to the IPs where the phase advances between two consecutive BPMs are far from $\frac{\pi}{2}$.

A comparison between the two approaches of paring the BPMs is given in Table I. The error bars for the measured data as well as for the simulated data are decreased by about a factor 2 .

\section{NOISE REDUCTION USING SINGULAR VALUE DECOMPOSITION}

SVD has been demonstrated to significantly reduce the noise in TbT data [4,29-31]. The TbT matrix (horizontal and vertical planes are separated) $A_{N \times M}$ where $M$ is the number of BPMs and $N$ is the number of turns recorded by each BPM can be decomposed according to

$$
A=U S V^{T},
$$

where $S_{N \times M}=\operatorname{diag}\left(\sigma_{1}, \ldots, \sigma_{m}\right)$ is a matrix containing the singular values, $U_{N \times N}$ is a matrix containing the temporal information and $V_{M \times M}$ contains the spatial information.

Each temporal mode is associated with a spatial mode representing the variation of the temporal mode along the machine. The magnitude of the singular value is representing the overall amplitude of the mode.

For BPM $m$ the TbT data: $b$ is fully reconstructed from the following equation

$$
\left(\begin{array}{c}
b_{1 m} \\
\vdots \\
b_{N m}
\end{array}\right)=\sum_{i=1}^{\mathrm{Opt}} \sigma_{i} v_{m i}\left(\begin{array}{c}
u_{1 i} \\
\vdots \\
u_{N i}
\end{array}\right),
$$

where $v_{m i}$ are the matrix elements of $V$ and $u_{m i}$ are the matrix elements of $U$, given that $\mathrm{Opt}=M$, meaning that all modes are used. Normally, however, this is not the optimal in terms of reconstructing the optics parameters since only a few modes contain the relevant information about the coupling. If the sum is truncated at the right point, the modes that represent noise will be cut away while the modes containing the motion of the particles will be kept. The problem arises in the determination of the numbers of singular values that should be kept in order to best reproduce the coupling.

It was suggested in [31] that 4 modes are enough to reconstruct the coupling given an appropriate rotation of the singular values mode into the physical modes is performed.

A simulation consisting of tracking with PTC [28] for 1000 turns was performed to increase the understanding of how to select the number of singular values to use. A realistic coupling was introduced in the model using skew quadrupoles. The reference $f_{1001}$ are determined from 


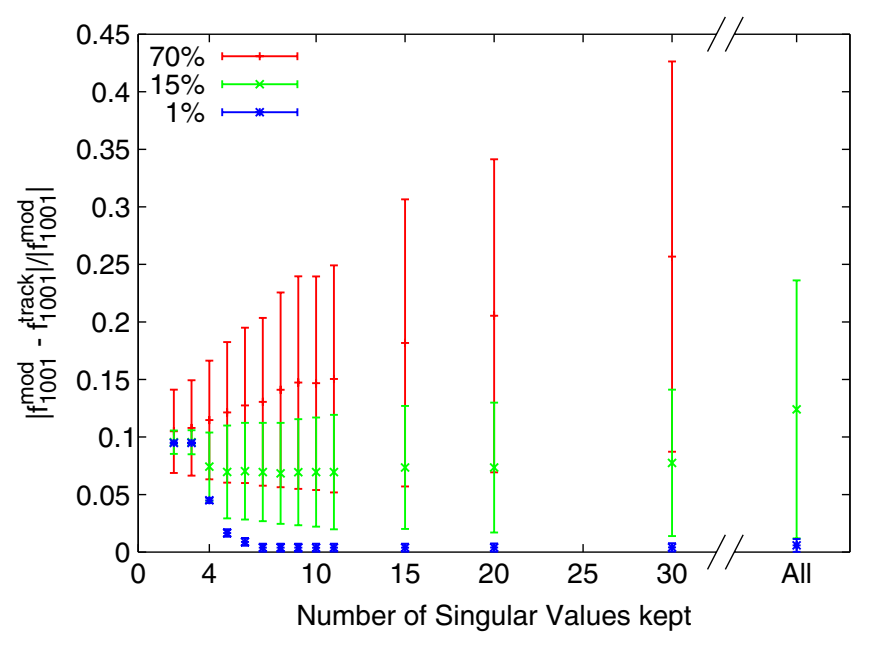

FIG. 3. The relative deviation from the correct coupling as a function of the number of singular values used. The error bars represent the average error bar of all the 502 simulated BPMs. When all singular values are used we are not able to calculate the coupling for the $70 \%$ noise case.

tracking without any noise and the use of all singular values. Gaussian noise of $1 \%, 15 \%$, and $70 \%$ was added to construct the $\mathrm{TbT}$ data used in the analysis. The deviations between the correct coupling and the reconstructed using different numbers of singular values are shown in Fig. 3. The error bars on the plot are the average statistical errors from all available BPMs. The statistical error bars are clearly decreasing with fewer singular values but the deviation from the correct value also increases for the lowest number of singular values, for the $1 \%$ and $15 \%$ noise cases. From this we conclude that reducing the number of singular values below 8 introduces a systematic error. Truncating at 4 singular values would introduce on average a 5\% error due to the mode mixing. The degree of mode mixing depends on the number of BPMs as well as on the number of modes: As a consequence 8 might not be the
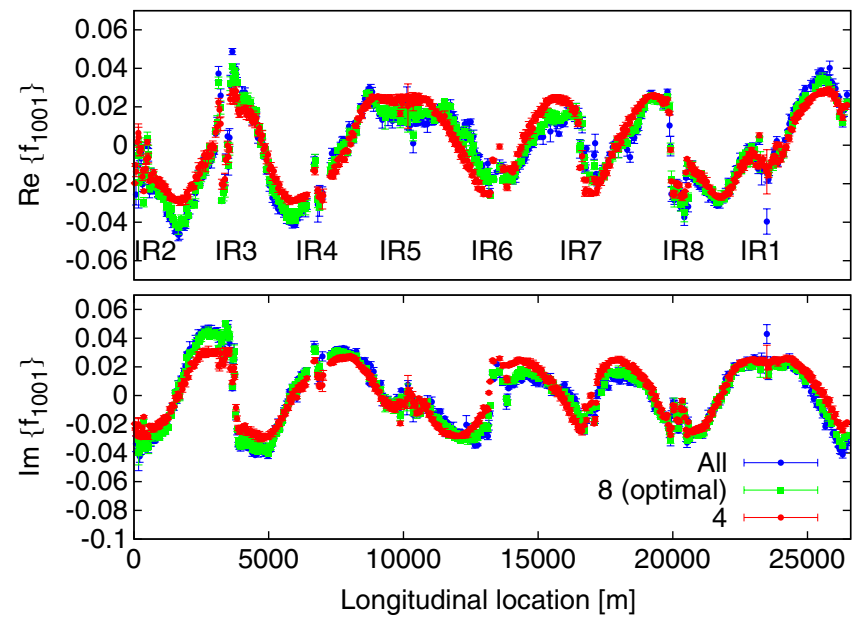

FIG. 4. The $f_{1001}$ reconstructed along the machine at injection for different number of singular values. optimal in other machines. The SVD cleaning will, however, reduce significantly the noise-induced error if applied correctly and for the $15 \%$ noise case the mean deviation from the correct value is reduced by a factor 1.85 when using 8 singular values compared to when no cleaning is performed.

In Fig. 4 the SVD cleaning is applied for data measured during the commissioning in 2012 for Beam 1. The noise levels in the LHC are usually around $10 \%-20 \%$ and as a consequence 8 singular values are the optimal choice based on our simulations. We observe that using only 4 singular values the coupling amplitude looks almost completely constant along the machine. This artefact is not present when the optimum 8 singular values are kept.

\section{AUTOMATIC CORRECTION USING INJECTION OSCILLATIONS}

Before every LHC fill a low intensity bunch is injected to validate parameters such as tune, chromaticity, and coupling. The BPM acquisition system was modified in June 2012 to be able to record the free oscillations using all the available BPMs for 2000 turns at injection. From the $f_{1001}$ inferred at all BPMs, the optimum setting of the coupling knobs is calculated. The use of all BPMs ensures a setting that minimizes the coupling globally. A strong local coupling source will manifest itself as a jump in the $\left|f_{1001}\right|$ along the machine [32]. This should be visible from the software responsible for the correction and a dedicated local correction can be applied later. No strong local sources were observed after the initial commissioning in 2012 [9]. The correction algorithm, without the presence of strong local sources, is based on a response matrix inversion. The response matrix $\mathbf{R}$ is created using the MAD model. The matrix relates the $f_{1001}$ at the BPMs with setting of the two knobs as

$$
\mathbf{R} \Delta \vec{K}_{\text {knobs }}=\left(\operatorname{Re}\left\{\vec{f}_{1001}\right\}, \operatorname{Im}\left\{\vec{f}_{1001}\right\}\right),
$$

where $\vec{f}_{1001}$ indicates that all available BPMs are used. The measured coupling is then multiplied with the pseudo matrix: $\mathbf{R}^{-1}$ to calculate the optimum setting of the coupling knobs. To make the algorithm automatic and robust against BPM failures the 5\% measurements deviating most from the average $\left|f_{1001}\right|$ are removed before the correction is calculated.

$$
\Delta \vec{K}_{\text {knobs }}=\mathbf{R}^{-1}\left(\operatorname{Re}\left\{\vec{f}_{1001}\right\}, \operatorname{Im}\left\{\vec{f}_{1001}\right\}\right) .
$$

Vertical dispersion is not included in the correction algorithm since it is not a major concern for the LHC and the correction knobs only marginally affect it.

Figure 5 shows data for 40 injections that were acquired over $6 \mathrm{~h}$. The blue stars show the settings of the coupling knobs that best reproduce the measurement in the model calculated from Eq. (8). Before three injections, marked 

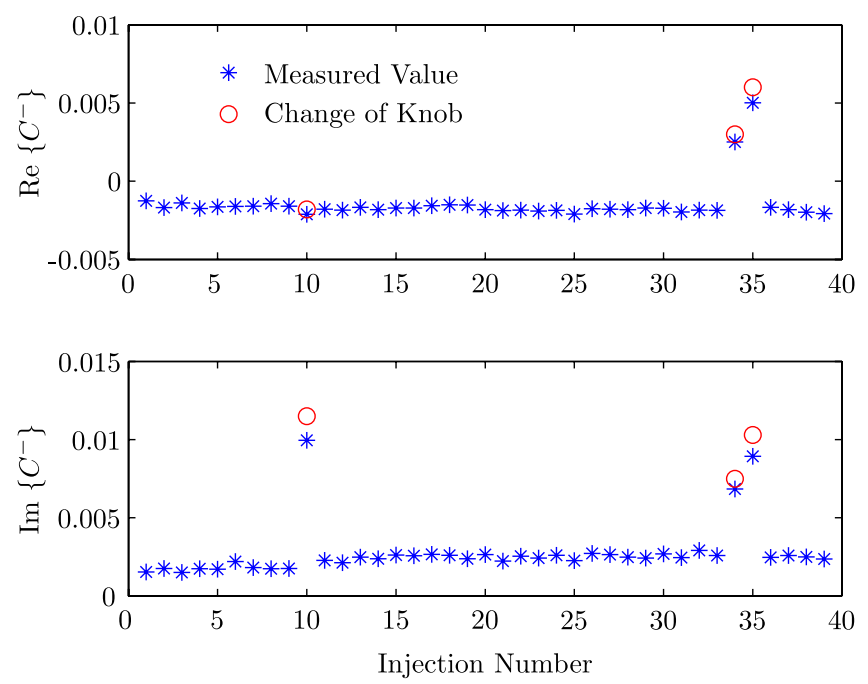

FIG. 5. Measured $C^{-}$for different injections during $6 \mathrm{~h}$. The blue stars shows the setting, in the model, of the coupling knobs which best reproduce the measured coupling. The red circles show the strength of the three manual changes that were applied.

with red circles in the figure, the settings of the coupling knobs were changed voluntarily. The circles indicate the values we expect to measure based on our model. The agreement between the predictions and measurements is good. We observe a small discrepancy, more pronounced for larger changes of the coupling knobs, between the measured $C^{-}$(as characterized in the plot by the equivalent LHC coupling knob settings-blue stars) and the applied knob settings (red circles). The effect however is small, and is not an obstacle in the use of these observables and knobs for correction. We can also conclude that the coupling remains stable on time scales of hours. This is an important observation since if the coupling changed in short time
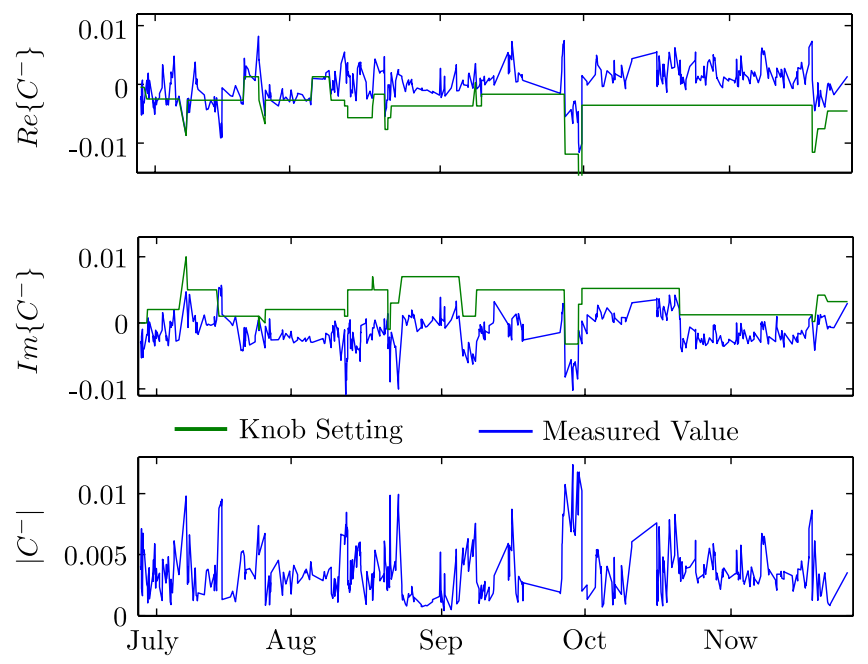

FIG. 6. The variation of the $C^{-}$from July 2012 until December 2012. The green lines shows the setting of the coupling knobs used and the blue shows the measured.
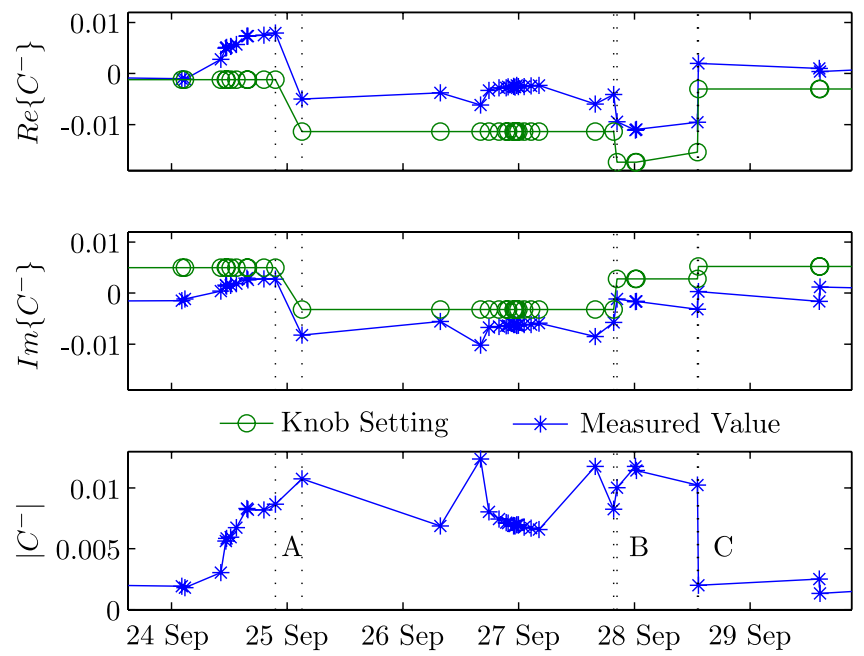

FIG. 7. The $C^{-}$after a technical stop. The green circle shows the setting of the correction knobs while the blue stars show the measured $C^{-}$. At A and B the coupling was adjusted manually, observing an increase in the coupling and at point $C$ the automatic correction was used, which successful decreased the coupling.

period then it would drift during the injection process which takes around 30 minutes.

Figure 6 shows the variation of the coupling in the LHC from July 2012 until the end of November 2012. The green line shows the setting of the coupling knobs while the blue shows the measured values. The $\left|C^{-}\right|$is calculated from Eq. (2) and the real and imaginary is obtained through fitting the setting of the knobs that best reproduce the measurements. We observe that the coupling is well corrected for most injections but on the time scale of a week it is necessary to perform a correction. In the presented time period most of the corrections were performed manually.

Figure 7 shows the coupling evolution during 5 days in the end of September. At point A it was observed that the coupling was a bit high and there was an attempt to correct it manually. As we can see the correction was unsuccessful and resulted in a higher $\left|C^{-}\right|$than before the correction. Between $\mathrm{A}$ and $\mathrm{B}$ there was a drift of the $C^{-}$. A new correction was attempted at point $\mathrm{B}$ which improved the situation for the imaginary part but made the situation worse for the real part and as a result the $\left|C^{-}\right|$increased slightly. At point $\mathrm{C}$ the method based on the injection oscillations was used to find the optimal setting of the coupling knobs. As seen in Fig. 7 the automatic correction successfully corrected the coupling. This example illustrates the benefits of the automatic correction procedure based on all available BPMs compared to the use of a single BPM.

\section{COUPLING FEEDBACK FOR THE LHC}

We observed in Sec. V that manual correction based on measurement from a single location is not always 
successful in correcting the coupling in the LHC. Ideally we would like to have automatic correction using as much information around the ring as possible. The measurement and correction procedure we have developed is based on a new type of high resolution BPM electronics, DOROS, that will be installed in the LHC [21]. At the initial stage 10 BPMs per beam will be equipped with the DOROS electronics but depending on the needs and the performance of the system more BPMs may be equipped later. BPMs equipped with this electronics will only need an excitation in the order of $10 \mu \mathrm{m}$ compared to almost $1 \mathrm{~mm}$ needed for the normal BPM system [21]. The phase advance between two BPMs equipped with this electronics are in most cases close to $\pi$ or 0 . As a consequence it is not suitable to use a coupling reconstruction algorithm based on 2 BPMs. However, using the amplitude and phase information from the tune and the coupling lines we can approximate the $f_{1001}$ from a single BPM [33]. The limitation is that it is no longer possible to separate the $f_{1001}$ from the $f_{1010}$. Figure 8 shows the simulated reconstructed coupling at the foreseen location for the system. We observe in Fig. 8 that the measurements are scattered around the correct value because of the contribution of the $f_{1010}$ to the $f_{1001}$.

In order to evaluate the impact of using single BPMs, a simulation was performed. A coupling situation based on a measurement in 2012 was introduced in the model. A correction was then calculated, based on 10 independent BPMs, at the locations foreseen for the BPMs equipped with the DOROS electronics, and for a BPM pair with a phase advance close to $\frac{\pi}{2}$. Figure 9 shows the comparison between using the system with two BPMs with a phase advance close to $\frac{\pi}{2}$ compared to the system where 10 BPMs independently measure the coupling. The comparison with a single BPM pair was done since this was also considered initially. For each noise level 50 data sets were generated

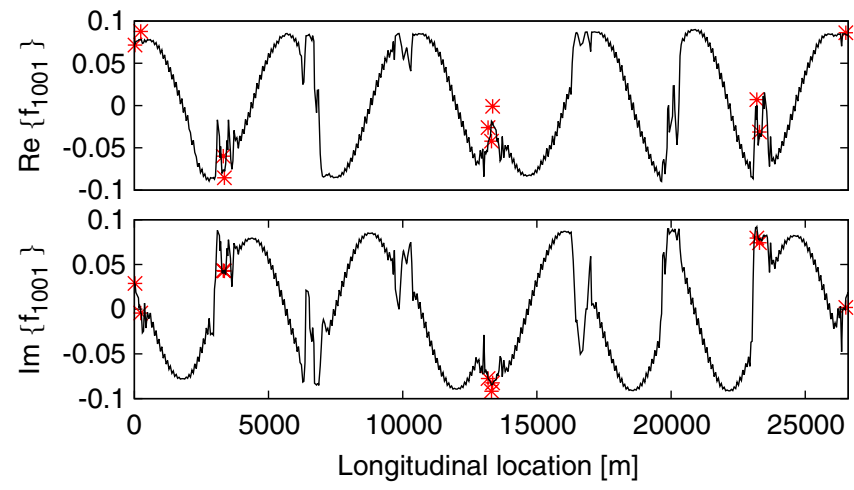

1 BPM Method $\quad *$

Model

FIG. 8. Simulated coupling along the machine. The red markers indicate the reconstructed coupling using the $1 \mathrm{BPM}$ method at the foreseen locations for the BPMs equipped with the DOROS electronics.

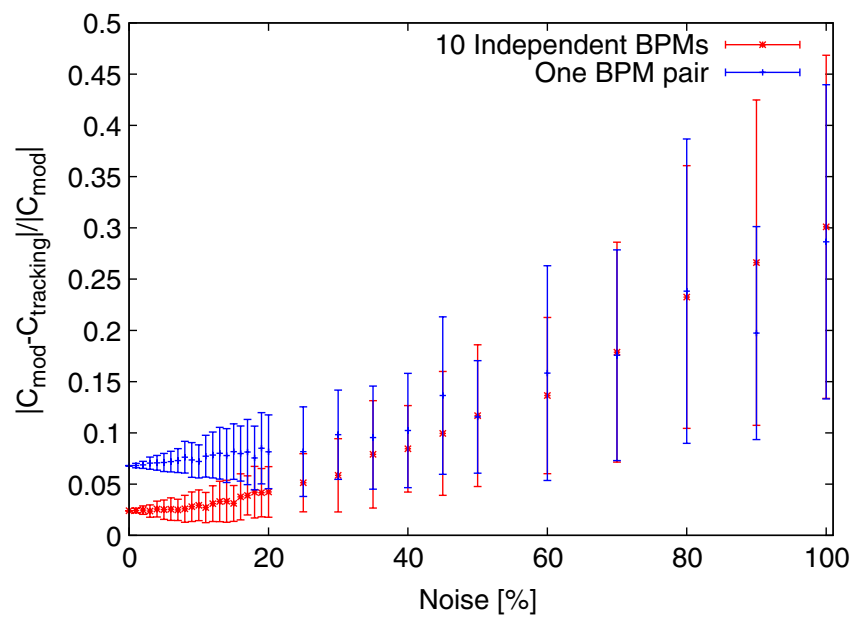

FIG. 9. The simulated performance of a system using several BPMs but only with the 1BPM method compared to a two BPMs at a single location separated with a phase advance of $\frac{\pi}{2}$.

and a correction was calculated for each of them. The error bar is the $\sigma$ of the calculated correction.

Although the 2 BPM method almost measures the coupling perfectly at a single location, when there is no noise, it still results in a less accurate coupling correction. The reason is that the coupling is varying slightly along the machine which is detected in case you use several BPMs. The error introduced by using 1 BPM reconstruction is dependent on the relative phase between the $f_{1001}$ and the $f_{1010}$. However, since we use several BPMs with different relative phases between $f_{1001}$ and the $f_{1010}$ the error averages out to a large extent. The signal-to-noise ratio of the measurement will depend on the amplitude of the excitation, the number of turns recorded and the final performance of the system. With an excitation of $10 \mu \mathrm{m}$ the anticipated noise level is around $10 \%$ which according to our simulations is an acceptable level for a good measurement of the coupling.

\section{CONCLUSION}

In this article we have presented the recent improvements in measuring and correcting the coupling in the LHC. An approach to measure and correct the coupling automatically, based on the free injection oscillations, has been shown. It has been demonstrated during normal LHC operation. The method benefits from the presented improvements in selecting pairs of BPMs with a phase advance close to $\frac{\pi}{2}$. The improvement with such an algorithm has been thoroughly demonstrated by simulation studies and with real data. The TbT data quality is also enhanced from the singular values noise reduction techniques described. In particular we have demonstrated the need to filter the data using 8 singular values for a precise coupling measurement in the LHC. These improvements have resulted in an increased resolution of the coupling with about a factor 3 . 
We have also shown the use of a more accurate formula relating the $f_{1001}$ to the $C^{-}$.

The layout of a new feedback for coupling correction based on a new type of high resolution BPM electronics, DOROS [21], which will be in operation in 2015 has been described. The system benefits from coupling acquisition from several locations around the machine. It has been demonstrated that even though the system will not be able to separate the $f_{1001}$ from the $f_{1010}$, it will be able to correct the global coupling.

\section{ACKNOWLEDGMENTS}

A big thanks to Andrea Franchi for testing and interpreting the improved relation between $f_{1001}$ and $C^{-}$and for valuable comments on the manuscript. Many thanks also to Marek Gasior and Jakub Olexa for providing crucial information about DOROS and for useful discussions. The authors are also grateful to thank Verena Kain and Delphine Jacquet for making the modifications which enabled all BPMs to record the injection oscillations. Thanks also to the entire Optics Measurement and Correction (OMC) team for their help during tests as well as for fruitful discussions. A special thanks to Yngve Levinsen for proofreading and to Ewen Maclean for allowing us to change the coupling during the nonlinear machine development and for fruitful discussions. Thanks also to Massimo Giovannozzi and Oliver Brüning for proofreading the manuscript. Finally, we would like to thank the LHC operation group for their assistance and in particular we would like to thank Giulia Papotti and Alick Macpherson for their help during the tests.

\section{APPENDIX}

The Resonance Driving Term (RDT) at location $b$ is related to the Hamiltonian terms as

$$
f_{j k l m}^{(b)}=\frac{\sum_{w} h_{w, j k l m} e^{-i\left[(j-k) \Delta \phi_{w, x}^{b}+(l-m) \Delta \phi_{w, y}^{b}\right]}}{1-e^{-2 \pi i\left[(j-k) Q_{x}+(l-m) Q_{y}\right]}},
$$

where the sum is over the corresponding elements and $\Delta \phi_{w}^{b}$ is the phase advance between the $w^{t} h$ multipole and the location $b$. Note that the sign of the phase in the exponential in Eq. (A1) varies across literature.

It was shown in [34] that RDTs amplitude remains constant in sections free of multipoles and shows abrupt jumps at the locations of these sources. The analytical expression describing these abrupt changes was given in [35] and [32] as

$$
\begin{aligned}
f_{j k l m}^{(2)}= & e^{-i\left[(k-j) \Delta \phi_{x}+(m-l) \Delta \phi_{y}\right]}\left[f_{j k l m}^{(1)}\right. \\
& \left.-\sum_{q=1}^{n} e^{i(k-j) \phi_{x q}+i(m-l) \phi_{y q}} h_{q, j k l m}\right],
\end{aligned}
$$

where $f_{j k l m}^{(2)}$ are the RDTs at a second location, $\Delta \phi_{x, y}$ are the horizontal and vertical phase advances between the two locations, the summation extends only over the multipoles placed in between the two locations, $\phi_{x q, y q}$ are the phase advances between the first location and the $q^{\text {th }}$ multipole, and $h_{q, j k l m}$ are real quantities proportional to the integrated strength of the $q^{\text {th }}$ multipole and to the product $\beta_{x q}^{\frac{j+k}{2}} \beta_{y q}^{\frac{l+m}{2}}$.

In the limit that $s_{2}$ tends to $s_{1}, \phi_{x q}$ and $\phi_{y q}$ tend to zero, Eq. (A2) takes the following differential form

$$
\begin{aligned}
\frac{d f_{j k l m}}{d s}= & -i\left[(k-j) \frac{d \phi_{x}}{d s}+(m-l) \frac{d \phi_{y}}{d s}\right] f_{j k l m}(s) \\
& -k_{j k l m}(s),
\end{aligned}
$$

where $f_{j k l m}(s)$ is now a continuous function of $s$ and $k_{j k l m}(s)$ is the corresponding local multipolar strength at $s$ (not integrated). It is convenient to introduce $A_{j k l m}(s)$ which we define as

$$
f_{j k l m}=A_{j k l m} e^{-i\left[(k-j) \phi_{x}(s)+(m-l) \phi_{y}(s)\right]} .
$$

Inserting Eq. (A4) into Eq. (A3) yields

$$
\frac{d A_{j k l m}}{d s}=-e^{i\left[(k-j) \phi_{x}(s)+(m-l) \phi_{y}(s)\right]} k_{j k l m}(s) .
$$

The relation between $k_{1001}$ and $C^{-}$is described in [10,22]

$$
C^{-}=\frac{4}{2 \pi} \oint d s k_{1001} e^{-i\left(\phi_{x}-\phi_{y}\right)+i s \Delta / R},
$$

where $\Delta$ is the fractional part of $Q_{x}-Q_{y}$. From Eq. (A5)

$$
\frac{d A_{1001}}{d s}=-e^{-i\left(\phi_{x}-\phi_{y}\right)} k_{1001}(s),
$$

therefore

$$
C^{-}=-\frac{4}{2 \pi} \oint d s \frac{d A_{1001}}{d s} e^{i s \Delta / R}
$$

Integration by parts yields

$$
C^{-}=-\frac{4}{2 \pi}\left[\left.A_{1001}(s) e^{i s \Delta / R}\right|_{0} ^{c}-\frac{i \Delta}{R} \oint d s A_{1001} e^{i s \Delta / R}\right] .
$$

The first term in the square brackets cancels out and Eq. (A4) is used to express $C^{-}$as a function of $f_{1001}$,

$$
C^{-}=\frac{4 \Delta i}{2 \pi R} \oint d s f_{1001} e^{-i\left(\phi_{x}-\phi_{y}\right)+i s \Delta / R} .
$$


[1] O. S. Brüning and S. D. Fartoukh, CERN-LHC-ProjectReport-501, 2001.

[2] R. Tomás, O. Brüning, M. Giovannozzi, P. Hagen, M. Lamont, F. Schmidt, G. Vanbavinckhove, M. Aiba, R. Calaga, and R. Miyamoto, Phys. Rev. ST Accel. Beams 13, 121004 (2010).

[3] R. Tomás, T. Bach, R. Calaga, A. Langner, Y. I. Levinsen, E. H. Maclean, T. H. B. Persson, P. K. Skowronski, M. Strzelczyk, G. Vanbavinckhove et al., Phys. Rev. ST Accel. Beams 15, 091001 (2012).

[4] S. White, E. Maclean, and R. Tomás, Phys. Rev. ST Accel. Beams 16, 071002 (2013).

[5] T. H. B. Persson, Y. Inntjore Levinsen, R. Tomás, and E. H. Maclean, Phys. Rev. ST Accel. Beams 16, 081003 (2013).

[6] E. H. Maclean, R. Tomás, F. Schmidt, and T. H. B. Persson (to be published).

[7] J. Serrano and M. Cattin, Technical note: CERN-BE-Note2010-014.

[8] R. Miyamoto, M. Aiba, R. Calaga, R. Tomás, and $\mathrm{G}$. Vanbavinckhove, in Proceedings of the 2nd International Particle Accelerator Conference, San Sebastián, Spain (EPS-AG, Spain, 2011).

[9] R. Tomas, T. Bach, P. Hagen, A. Langner, Y. Levinsen, M. McAteer, E. Maclean, T. Persson, P. Skowronski, and $\mathrm{S}$. White, in Proceedings of the LHC Beam Operation Workshop-Evian (CERN, Evian, France, 2012).

[10] G. Ripken and F. Willeke, Part. Accel. 27, 203 (1990).

[11] R. Tomas, "Optimizing the Global Coupling Knobs for the LHC" (unpublished); http://cds.cern.ch/record/1422434.

[12] A. Boccardi, M. Gasior, R. Jones, P. Karlsson, and R. Steinhagen, CERN-LHC-Performance-Note-007, 2009.

[13] M. G. Minty and F. Zimmermann, Measurement and Control of Charged Beams (Springer, New York, 2003).

[14] W. Daqa, V. Kornilov, I. Hofmann, and O. BoineFrankenheim, "Linear Betatron Coupling in SIS-18", https://www-alt.gsi.de/documents/DOC-2009-Oct-250-1 .pdf.

[15] W. Fischer, Phys. Rev. ST Accel. Beams 6, 062801 (2003).

[16] M. Benedikt, F. Schmidt, R. Tomás, P. Urschütz, and A. Faus-Golfe, Phys. Rev. ST Accel. Beams 10, 034002 (2007).
[17] Y. Luo, P. Cameron, A. Della Penna, J. Laster, R. Lee, A. Marusic, F. Pilat, T. Roser, D. Trbojevic, and J. Wei, Phys. Rev. ST Accel. Beams 8, 014001 (2005).

[18] Y. Luo, P. Cameron, A. Della Penna, J. Laster, A. Marusic, F. Pilat, T. Roser, and D. Trbojevic, Phys. Rev. ST Accel. Beams 8, 074002 (2005).

[19] P. Cameron, A. DellaPenna, L. Hoff, Y. Luo, A. Marusic, C. Schultheiss, S. Tepikian, M. Gasior, R. Jones, and C. Y. Tan, Phys. Rev. ST Accel. Beams 9, 122801 (2006).

[20] R. Calaga, R. Tomás, and A. Franchi, Phys. Rev. ST Accel. Beams 8, 034001 (2005).

[21] J. Olexa, O. Ondracek, Z. Brezovic, and M. Gasior, CERN Report No. CERN-ATS-2013-038, 2013.

[22] A. Franchi, Ph.D. thesis, GSI DISS (2006) [http://www-alt .gsi.de/documents/DOC-2007-Jun-38-1.pdf].

[23] Y. Alexahin and E. Gianfelice-Wendt, JINST 6, P10006 (2011).

[24] MAD-X, MAD-X homepage, [online]: http://madx.web .cern.ch/madx/.

[25] A. Franchi (private communication).

[26] R. Bartolini and F. Schmidt, Part. Accel. 59, 93 (1997).

[27] D. D. Caussyn, M. Ball, B. Brabson, J. Collins, S. A. Curtis, V. Derenchuck, D. DuPlantis, G. East, M. Ellison, T. Ellison et al., Phys. Rev. A 46, 7942 (1992).

[28] F. Schmidt, E. Forest, and E. McIntosh, CERN Report No. CERN-SL-2002-044-AP; Report No. KEK-REPORT2002-3, 2002.

[29] R. Calaga and R. Tomás, Phys. Rev. ST Accel. Beams 7, 042801 (2004).

[30] G. Vanbavinckhove, Ph.D. thesis, FNWI: Institude of Physics (IOP), 2013 [http://dare.uva.nl/record/434477].

[31] C.-x. Wang, Phys. Rev. ST Accel. Beams 7, 114001 (2004).

[32] A. Franchi, R. Tomás, and F. Schmidt, Phys. Rev. ST Accel. Beams 10, 074001 (2007).

[33] A. Franchi, L. Farvacque, F. Ewald, G. L. Bec, and K. Scheidt, arXiv:1402.1461.

[34] R. Tomas-Garcia, Ph.D. thesis, Valencia U. València, 2003.

[35] R. Tomás, M. Bai, R. Calaga, W. Fischer, A. Franchi, and G. Rumolo, Phys. Rev. ST Accel. Beams 8, 024001 (2005). 